\title{
Les chemins d'une professeure conceptrice-lumière
}

\section{Os caminhos de uma iluminadora-professora}

The paths of an lighting designer teacher

Entretien accordé par Véronique Perruchon à Priscila Costa ${ }^{1}$ et à José Ronaldo Faleiro ${ }^{2}$ 


\section{Résumé}

Cet entretien a été accordé par Véronique Perruchon à Priscila Costa et à José Ronaldo Faleiro, lors de l'évément $A$ Luz em Cena, qui s' est tenu du 2 au 6 septembre 2019 au Centre des Arts (CEART) de l'Université d'État de Santa Catarina (UDESC), au Brésil. Dans un discours passionné et didactique, Véronique Perruchon expose des données sur l'enseignement, la formation et la pratique des éclairagistes en France, du milieu du XXe siècle à nos jours. Elle décrit avec clarté sa recherche d'une dimension sensible dans la relation entre la lumière et le noir.

Mots-clés: Formation d'éclairagistes ; lumière scénique et philosophie ; lumière et noir.

\section{Resumo}

Esta entrevista foi concedida por Véronique Perruchon a Priscila Costa e a José Ronaldo Faleiro, durante o evento A Luz em Cena, realizado de 02 a 06 de setembro de 2019 no Centro de Artes (CEART), da Universidade do Estado de Santa Catarina (UDESC), Brasil. Com precisão e por vezes lirismo, Véronique Perruchon expõe dados sobre o ensino, sobre a formação e sobre a prática de iluminadoras e iluminadores na França, desde a metade do século $X X$ até 0 presente. A entrevistada relata com clareza a sua busca pela dimensão sensível da relação entre a luz e o escuro.

Palavras-chaves: Formação de iluminadores (as); iluminação e filosofia; luz e escuro.

\section{Abstract}

This interview was conducted in September 2019 by Priscila Costa and José Ronaldo Faleiro during the event A Luz em Cena held from the 2nd to the 6th of September 2019 at the Arts Center (CEART) of the Santa Catarina State University (UDESC) in Brazil. In a passionate and instructional speech, Véronique Perruchon exposes data on the teaching, training and practice of lighting designers in France from the mid-twentieth century to this day. The interviewee gave a clear review of her research on the sensitive dimension of the relationship between light and dark.

Keywords: Lighting design courses; lighting and philosophy; light and dark.

E-ISSN: 2358.6958

\footnotetext{
${ }^{1}$ Eclairagiste et étudiante en Master Arts de la scène et du spectacle vivant - Université d'Artois, France. priscilacoast@gmail.com

${ }^{2}$ Docteur en Arts du Spectacle de l'Université Paris X - Nanterre. Professeur chercheur au Centre des Arts de l'Université d'État de Santa Catarina (UDESC). irfalei@gmail.com
} 


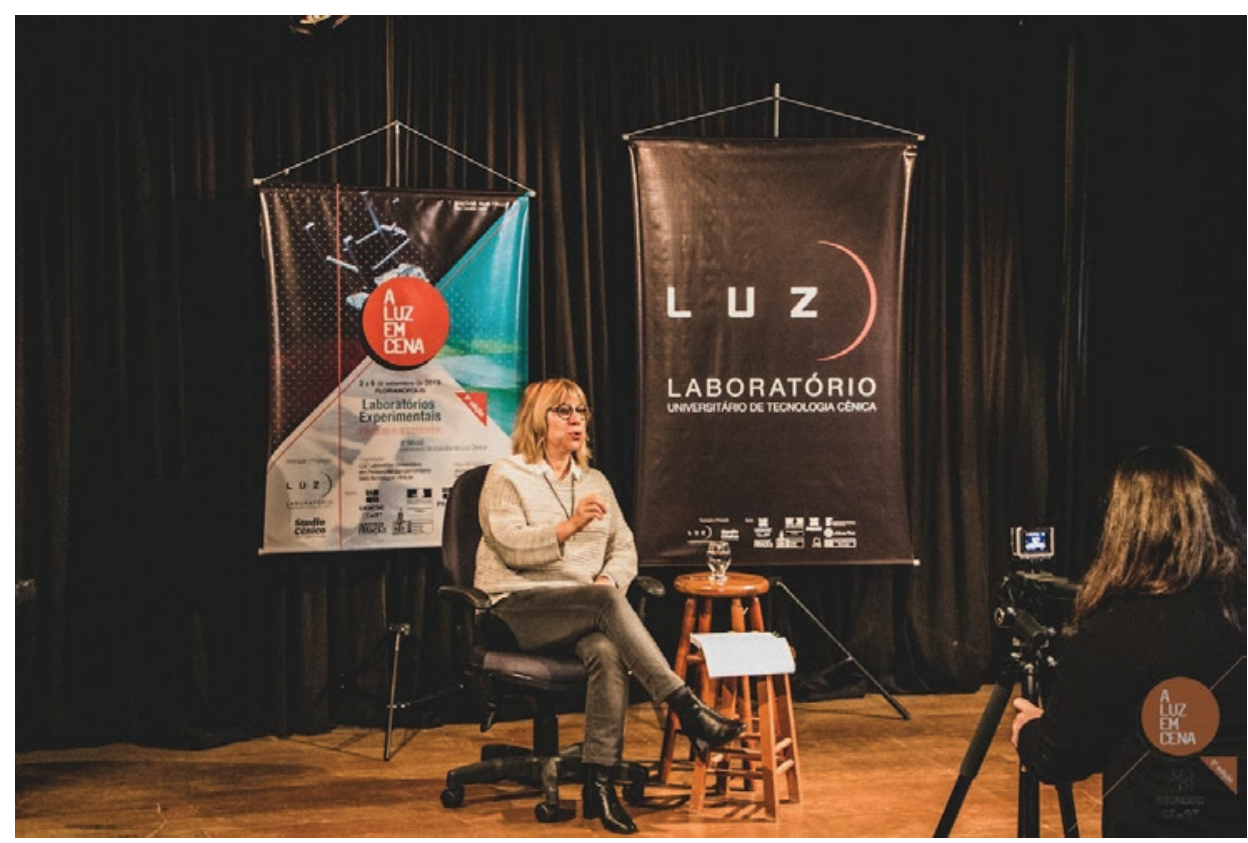

Véronique Perruchon.

Professeure en Arts de la Scène à l'Université de Lille et membre du Centre d'Études des Arts contemporains, dirige un programme de recherche sur la « Lumière de Spectacle ». Après des débuts professionnels en tant qu'éclairagiste et régisseuse, elle s'est intéressée au domaine spectaculaire du théâtre et à la mise en scène dans son travail de recherche. Elle publie aux Presses universitaires du Septentrion, les ouvrages : Noir. Lumière et théâtralité, en 2016, et André Engel. Fuvre théâtrale, en 2018. Références intitutionnelles : LdS Programme de recherche « Lumière de Spectacle », Université de Lille. EA 3587 - CEAC - Centre d'Étude des Arts Contemporains. 59000 Lille, France. 
José Ronaldo Faleiro et Priscila Costa - Merci beaucoup de nous accorder cet entretien.

Faleiro - Vous avez écrit votre thèse sur le metteur en scène André Engel. Pourrait-on voir là un intérêt pour le théâtre fait à l'Est de la France?

Pas spécialement. On ne peut pas dire qu'il y ait une régionalisation des manières de faire du théâtre en France. En revanche, dans les années 70, à l'époque où André Engel a commencé à faire du théâtre au Théâtre Nationale de Strasbourg (le TNS), il y avait effectivement une spécificité qui était due à cet endroit et à la direction de Jean-Pierre Vincent. Il y avait la compagnie fondée par Jean-Pierre Vincent et Jean Jourdheuil, appelée compagnie Vincent-Jourdheuil. Ils avaient choisi de s'intéresser au modèle allemand. À savoir qu'ils ont choisi de faire des productions qui n'étaient pas créées par un seul metteur en scène mais par une alternance d'équipe qu'ils appelaient les "teams". Ces "teams" étaient composées d'au moins deux personnes qui pouvaient être quelqu'un qui devenait metteur en scène, ou régisseur à la manière allemande, avec l'autre qui était dramaturge. Ces personnes pouvaient être un metteur en scène, un scénographe, voire des associations diverses.

\section{Faleiro - Et quelle y était la place de l'éclairage?}

Ils ont invité André Diot à travailler avec eux. André Diot venait du cinéma, il était chef opérateur, ce qui veut dire qu'il s'occupait de la lumière du cinéma et il a apporté les outils du cinéma. Alors pourquoi? Quand André Engel a commencé à faire du théâtre, il a rencontré durant l'été 1969 une équipe qui faisait du cinéma, sur le tournage d'un film dans les Cévennes, Les Camisards (1972), sous la direction de René Allio. Engel était là un peu par hasard. Il avait fait des études de philo, il avait enseigné la philo un peu, dans un lycée, et il y était, là, comme pas mal de jeunes... on va dire hippies de l'époque. Il y avait le tournage de ce film et il y a fait de la figuration, mais en fait pendant les séances de débriefing et de travail autour du film, il a commencé à tenir des propos qui ont beaucoup intéressé Jean-Pierre Vincent et l'équipe. Donc il les a rejoints à Strasbourg et il a commencé à faire du théâtre mais il n'a jamais fait, à ce moment-là, de théâtre dans les salles. Il a immédiatement pensé à faire du théâtre hors les murs. C'est-à-dire dans des espaces choisis qui ne sont pas ceux du théâtre. On peut comprendre l'importance que cela pouvait avoir pour André Diot, qui venait du cinéma, c'est-à-dire qui était habitué à éclairer les espaces naturels. Je pense que la filiation vient par le cinéma et la transposition du théâtre dans des espaces naturels.

Faleiro - Vous avez écrit que André Engel dans les années 70-80 était connu dans son travail, avec l'éclairagiste André Diot justement, pour mettre le spectateur mal à l'aise, parce qu'il enlevait le confort de la visibilité du plein feu, pour que le spectateur fasse un effort de voir et d'entendre. Est-ce que vous voyez aujourd'hui des créateurs qui suivent ce même chemin ou ce même procédé? 
Alors, l'idée de mettre le spectateur mal à l'aise c'était d'une part les fondamentaux d'une réflexion de l'Internationale situationniste et de Guy Debord, qui critiquaient la Société du Spectacle. Donc, il y avait l'idée de critiquer la société du spectacle à travers des spectacles qui renforçaient la position du spectateur. Les gens devenaient un "spectateur amplifié". Pas au sens où nous l'entendons maintenant avec les techniques d'amplification, mais il était obligé d'être spectateur de façon accrue. Soit par la situation dans laquelle on le mettait, c'est-à-dire qu'il pouvait devenir le badaud d'un événement. Au festival de Nancy, dans la nature, sur une mine désaffectée pour Prométhée porte-feu (1980), le public assistait à l'arrestation d'un forcené avec un hélicoptère qui arrivait, avec les policiers, les pompiers... Ça durait trente minutes et c'est tout. Donc, ça pouvait être : le mettre dans une situation particulière de spectateur pour se poser des questions sur ce que c'est que d'être là et de voir la vie en train de se faire. Mais il y avait aussi un fond politique, un engagement à mettre mal à l'aise le spectateur pour qu'il s'interroge lui-même sur sa position. Maintenant, on voit des spectacles qui mettent le spectateur mal à l'aise, ça a été le cas notamment avec des spectacles très violents, très dérangeants, scatologiques comme ceux de Rodrigo Garcia, par exemple, ou du flamand Jan Fabre. La démarche est différente. Il n'y a pas la même réflexion philosophique, ou en tous les cas les racines de la réflexion philosophique ne sont pas les mêmes. Du tout. L'idée, pour Rodrigo Garcia je me souviens l'avoir lu dans les années 90, quand il a commencé à être connu en France, il parlait du spectateur endormi qu'il fallait réveiller. Bien sûr, il y a là une filiation brechtienne, peut-être mâtinée d'une influence du Théâtre de la cruauté d'Artaud, mais tout cela revisité parce que Rodrigo Garcia vient de la publicité. Donc, il utilisait le langage publicitaire, on va dire de la provocation, de la séduction pour les dénonciations des travers de la société qu'il voulait présenter sur scène. Donc c'est une autre pensée.

Ensuite, il y a des spectacles qui peuvent mettre le spectateur en difficulté de bien voir ou d'entendre mais pas du tout de la même manière qu'à l'époque d'André Engel et d'André Diot. Au contraire, en provoquant davantage un univers sonore très envahissant avec de la musique très forte et parfois avec des lumières qui sont au contraire de plus en plus lumineuses et fortes. J'ai pensé par exemple au spectacle $N$ (2004) d'Angelin Preljocaj, qui utilisait ces procédés, et même à d'autres spectacles qui ponctuellement à certains endroits vont utiliser ces principes-là. Une jeune génération va plutôt travailler dans ce sens-là.

\section{Faleiro - J'ai parfois l'impression que l'éclairagiste est au service du met- teur en scène. Est-ce qu'on pourrait imaginer une thèse dans laquelle l'éclaira- giste soit le protagoniste, l'objet de l'étude ? Est-ce que cela commence déjà à être fait, dans des groupes d'études?}

Je suis tout à fait d'accord avec ce propos. L'éclairagiste reste dans l'ombre, on n'en parle que secondairement et on pense qu'il est au service du metteur en scène. En réalité, les choses sont un peu différentes parce que soit il y a une association entre un metteur en scène et un éclairagiste qui travaillent dans la connivence et qui comprennent comment travailler ensemble (donc, ils sont d'accord sur la manière d'éclairer le spectacle), soit un metteur en scène va chercher un éclairagiste et va 
pouvoir dialoguer avec lui pour voir quel type d'éclairage il veut faire. Soit [le metteur en scène] tombe sur un éclairagiste qui effectivement va s'adapter à ses besoins, à ses désirs, soit il va tomber sur un éclairagiste qui peut infléchir le travail du metteur en scène en lui suggérant une autre manière de dire la même chose, avec un éclairage qui vient de sa propre sensibilité, ou encore carrément arriver avec sa propre esthétique et il sera choisi pour cette esthétique-là.

Elsa Revol par exemple a travaillé occasionnellement avec Wajdi Mouawad pour le dernier spectacle, Fauves (2019), et de fait la lumière est différente de ce que l'éclairagiste Éric Champoux faisait habituellement. Elle est arrivée aussi avec son esthétique. Et elle a fait la même chose avec Ariane Mnouchkine, c'est-à-dire que Ariane était de la génération du théâtre brechtien, avec une lumière de plein feu, on va dire, avec l'idée brechtienne que tout doit être vu, qu'il n'y a rien à cacher. Elle l'a accompagnée dans la possibilité d'un assombrissement et c'était fort à propos par exemple pour la pièce Macbeth (2014). De fait, on a une certaine obscurité sur le théâtre dans l'éclairage de la pièce Macbeth montée par Ariane Mnouchkine avec le Théâtre du Soleil. Ce qui est complètement inédit pour le Théâtre du Soleil.

Et pour la deuxième partie de la réponse, concernant les thèses possibles, pour l'instant on n'a pas encore de candidats pour faire une thèse sur un éclairagiste. Effectivement, parce que c'est très peu connu. J'espère qu'il y en aura.

\section{Faleiro - Mais croyez-vous qu'il y a de la documentation en France pour le faire?}

Ce n'est pas impossible, dans le sens où si c'est assez récent. On a beaucoup de photographies, de captations et de documents de notation. Il y a une documentation, mais elle n'est pas référencée sous cette appellation. C'est-à-dire que c'est en travaillant sur des spectacles qu'on va découvrir la documentation qui va nous intéresser. C'est tout à coup regarder les fonds documentaires existants sur les spectacles avec un nouveau regard : celui du chercheur qui s'intéresse spécifiquement à la lumière. C'est ce que j'ai déjà fait avec la documentation du fonds Jean Vilar qui est dans une antenne de la Bibliothèque Nationale de France (la BNF), à Avignon, et j'ai collecté effectivement un certain nombre de renseignements qui sont présents ici ou là mais qui ne sont même pas référencés comme étant des renseignements utiles sur la lumière.

\section{Faleiro - C'est précieux.}

Oui, mais je n'ai rien fait pour l'instant. J'ai simplement collecté.

\section{Faleiro - Et les théâtres, comme le TNS... est-ce qu'ils ont le plan de feu de leurs spectacles?}

Je ne pense pas que l'habitude ait été de garder les plans de feu. S'il y a des plans de feu ce sont ceux de l'éclairagiste lui-même dans le cas d'une reprise (donc il faudrait avoir la documentation des éclairagistes) et sauf peut-être dans le cas des théâtres qui proposent une alternance, comme précisément la Comédie-Française, où on est susceptible de reprendre un spectacle. Et voilà, vous me suggérez un tra- 
vail de recherche, parce qu'on pourrait très bien s'intéresser pour savoir quelle a été l'évolution de la lumière... si on a le plan de feu de la première fois et si ce fonds documentaire existe.

\section{Faleiro - Quelque chose d"écrit par les éclairagistes...}

André Diot n'a aucune trace écrite. C'est quelqu'un qui arrive et qui fait les choses en direct, encore maintenant. Et on respecte sa façon de travailler parce que c'est un grand éclairagiste. L'économie du spectacle vivant va très vite, maintenant. Il faut être rapide et efficace. Les temps de montage sont beaucoup plus courts dans le théâtre, donc il faut préparer en amont. Il faut préparer un plan de feu, etc., les choses sont beaucoup plus formalisées. Ce qui laisse très peu de place à une forme d'improvisation, et ça c'est un autre champ intéressant. Une question, peut-être : quelle sera la place de l'improvisation dans l'éclairage?

\section{Faleiro - Et comment on pourra l'enregistrer.}

Voilà. La question de la notation...

\section{Faleiro - Maintenant, on va peut-être la filmer, l'enregistrer de différentes manières. Si ce n'est pas par l'écrit, comment faire ? Mais n'y a-t-il pas une obliga- tion de laisser quelques traces dans les archives des théâtres... ?}

Ce n'est pas le cas actuellement. Mais il faut savoir que sur la période qui va de 1870 à 1945 il existe un fonds documentaire qui s'appelle ART (l'association des régisseurs de théâtre) et qui se trouve à la Bibliothèque Historique de la Ville de Paris. C'est remarquable, et il y a un site internet qui a été fait par Serge Bouillon et que son épouse, Danielle Mathieu-Bouillon, a perpétué, car il est décédé maintenant. Elle a été directrice de différents théâtres, dont le Théâtre Antoine dans sa dernière carrière, assez récemment, et qui est devenu un théâtre privé en France.

Il y a donc des fonds documentaires et j'ai pensé dans un premier temps que le terme régisseur de théâtre signifiait régisseur-lumière, régisseur-scénique... En fait, il s'agirait plutôt du terme allemand le Regisseur, c'est-à-dire le metteur en scène. Ce sont des spectacles qui ont beaucoup tourné en France et donc nous en avons des traces et parfois des successions de traces de différentes époques ou dates auxquelles ces spectacles ont tourné avec peut-être cinq ou six manuscrits tapés à la machine, à l'époque, avec des indications sur le son, sur les déplacements, avec des schémas pour les déplacements, parfois quelques photos (au début du vingtième siècle on avait quelques photographies des spectacles), quelques schémas... Le fonds est énorme. Donc il faut chercher et essayer. Il a été répertorié par des universitaires, mais parfois quand on a l'indication d'une lampe c'est marqué lumière, donc on se précipite si on est intéressé et en fait on nous dit que dans les accessoires, il y a un abat-jour. C'est une indication si on veut, pour la lumière, mais ce n'est pas une indication que, de notre point de vue du XXle siècle, nous pensions trouver.

Parfois, on trouve des choses très intéressantes et j'ai pu travailler notamment sur une pièce qui a été créée en 1929 au Théâtre du Gymnase à Paris, qui a énormé- 
ment tourné et qui s'appelle Mélo, de Henri Bernstein. Cette pièce a eu un tel succès que les documents sont très précis et j'ai pu reconstituer la quantité de lumière, la manière dont cette lumière était produite et son évolution d'une scène à l'autre. Et ce spectacle, ce scénario a eu un tel succès qu'Alain Resnais en a fait un film (en 1986) qui est tourné comme s'il était dans un théâtre. Le film commence par l'ouverture d'un rideau, on entend le bruissement de la salle, alors que la première mise en scène par Henri Bernstein de cette pièce était très influencée par une sorte de montage cinématographique.

\section{Faleiro - Et la collection Rondel ? Vous croyez qu'on pourrait y trouver des choses, là aussi ?}

Oui, mais je pense qu'avec la collection Rondel nous avons davantage affaire à une bibliothèque que de fonds documentaire. En revanche, à la Bibliothèque Nationale de France, il y a un fonds documentaire très intéressant qui s'appelle le fonds Clémançon. Clémançon était le nom de la société qui a fait le premier "jeu d'orgue" en France. Ils se sont occupés de l'installation du gaz dans les théâtres et on l'a appelé "jeu d'orgue" parce que la distribution du gaz par les tuyaux ressemblait à un orgue musical et que le jeu lui-même fonctionnait comme les touches d'un orgue avec le nom des tuyaux, et on pouvait ouvrir, fermer ou réduire le gaz. Ils ont été à l'origine de l'installation du gaz et ensuite ils se sont intéressés à l'équipement électrique dans les théâtres. Donc dans ce fonds, on a le nom des théâtres, on a des plaquettes de publicité de présentation de leur matériel, on a quelques articles qui parlent de leur équipement et de la manière dont ils ont équipé les théâtres, etc. J'ai eu la chance de regarder ces documents avant qu'ils ne soient organisés par les bibliothécaires. C'était plus facile à consulter, parce que je pouvais naviguer en fonction de mon propre intérêt. Maintenant, c'est organisé d'une manière académique qui n'est peut-être pas celle qui m'intéresserait. Donc, il est plus difficile de trouver la documentation ; il faudrait avoir toutes les boîtes à disposition pour les ouvrir.

\section{Faleiro - ... être un peu détective...}

Exactement, il faut être un peu détective et c'est très intéressant.

Faleiro - Vous avez parlé de la lumière parfois en rapport avec la philosophie, d'une certaine façon. Alors je vous demande quelles sont vos références, vos sources, vos lectures... Des images peut-être, ou des conférences... ce que vous entendez ou lisez etc... qui vous aident dans votre recherche?

Il y a deux ouvrages qui me semblent très importants, qui sont très poétiques tout en étant philosophiques et réflexifs, c'est La Flamme d'une chandelle (1961), de Gaston Bachelard, et L'Éloge de l'ombre (1933), de Junichirō Tanizaki. Ce sont deux ouvrages très importants pour moi, parce que la dimension sensible de la relation à la lumière et au noir est très importante. Je ne peux pas réfléchir en occultant la dimension sensible : elle est prioritaire chez moi. Je pars toujours du sensible. Donc, il n'est pas étonnant que je m'y sois intéressée. Bien sûr, je me suis intéressée aussi à la 
philosophie de Roland Barthes. Disons que ce qui m'intéresse c'est une sémiologie sensible chez Roland Barthes, où on sent la relation aux choses. Ce n'est pas inorganique et la relation organique est importante. Il est important pour moi de sentir la présence de celui qui voit, de celui qui reçoit : la subjectivité de la réception est très importante. Henri Bergson et la phénoménologie de la perception aussi vont dans ce sens. Ce sont des références pour moi. J'ai été très intéressée également, pour le travail sur André Engel mais aussi pour la lumière, par le rapport au cinéma selon Deleuze.

Actuellement je m'intéresse beaucoup aux écrits de Guattari, et spécifiquement à son travail sur l'écosophie. On trouve toujours ce rapport au monde, ce rapport sensible aux choses qui nous entourent et le rapport sensible à la lumière. Je n'ai pas un rapport détaché et objectif, pas du tout. Donc j'ai du mal à me retrouver dans l'école de Patrice Pavis.

Costa - A propos du contexte français, j'en profite pour vous demander quel est le statut aujourd'hui des éclairagistes, des créateurs lumière en France. Sontils considérés comme des artistes ? Vous en avez déjà parlé un peu plus tôt, quand vous évoquiez la relation avec le metteur en scène. Mais selon vous, comment les situeriez-vous?

Je vais répondre de trois manières différentes. La première, j'ai déjà un peu répondu, c'est le rapport entre les artistes eux-mêmes. Je pense qu'il y a une meilleure compréhension de la qualité artistique, de la spécificité esthétique et plus globalement de l'univers de l'éclairagiste. Ils sont de plus en plus en train de se faire connaître grâce aux sites internet. La plupart maintenant ont leur site, ce qui permet de sentir un peu mieux leur travail. Cependant, ça reste très confidentiel pour des gens qui s'intéressent à la lumière. La deuxième chose est complètement économique. L'identification d'un métier se lit sur la fiche de paie. Quel est l'intitulé de la fiche de paye et quelles en sont les répercussions sur le traitement économique par rapport au statut d'intermittent en France ? Je ne suis peut-être pas la meilleure personne pour répondre de façon pointue, mais je peux vous dire qu'ils ont été répertoriés comme techniciens. Ils ne peuvent donc pas être affiliés à la Maison des Artistes tant qu'ils sont identifiés comme techniciens. Certains commencent à faire valoir un statut d'auteur-créateur de lumière. Du point de vue de la cotisation, de la retraite, pour leur statut économique cette appellation est importante, mais c'est aussi un révélateur que de nommer les éclairagistes « créateurs lumière ». Le premier qui a eu cette reconnaissance c'est Pierre Savron, qui a travaillé avec Jean Vilar et qui l'a désigné notamment sur sa fiche de paye comme tel. C'était le premier à pouvoir être repéré comme un artiste technicien. C'est devenu technicien ensuite, mais finalement c'était déjà une reconnaissance.

Costa - Existe-t-il, en France, une différence de personnel ou de matériel, par exemple en ce qui concerne les projecteurs, entre les théâtres publics et les théâtres privés? 
Non, je crois qu'on a à peu près le même type de matériel, si ce n'est que le théâtre privé a peut-être plus de moyens financiers pour un équipement et sa rentabilité, et par le fait qu'il accueille autant de théâtre que de one-man shows et de shows. Ce qui a amené les théâtres privés à s'équiper de projecteurs automatiques et de leds avant les théâtres publics. Je dirais que c'est plutôt globalement entre le théâtre et la variété et les concerts qu'il y a des différences.

Mais il y a vraiment une évolution maintenant, puisqu'en France on ne peut plus utiliser les lampes à incandescence et les théâtres sont encouragés à utiliser les leds. Malheureusement, comme on le sait, un projecteur à led très performant coûte quatre fois plus cher qu'un PC. Donc cela pose un problème économique. On est exactement dans une situation transitoire. Mais il y a un théâtre à Montpellier qui s'est équipé entièrement aux leds et qui a décidé d'être un théâtre écologique.

\section{Costa - Est-ce que vous avez une méthodologie de l'enseignement de la lumière scénique ? Et que pensez-vous être indispensable dans la formation d'un éclairagiste?}

Que les choses soient claires par rapport à la France : à l'université on ne forme pas des éclairagistes. Vous appelez laboratoire ce qu'en France on appelle « ateliers de pratique ". J'ai ouvert un atelier de pratique avec une salle équipée de projecteurs, donc nous pouvons expérimenter et apprendre. De même que les étudiants ont une formation au jeu scénique, s'ils sont dans une école à côté ou s'ils estiment qu'ils sont suffisamment formés et souhaitent devenir des professionnels, personne ne va les en empêcher, bien évidemment, mais nous n'avons pas cette prétention. Si en sortant de la formation en éclairage de l'université de Lille, un étudiant décide tout de suite de devenir professionnel, il faut simplement qu'il continue à travailler pour apprendre tout seul, pour se perfectionner. En France, il y a des lieux précis, des écoles où on forme spécifiquement au métier de la lumière et de l'éclairage.

Alors je vais moins parler de la formation des éclairagistes peut-être, mais plutôt de la manière dont je les initie. On va parler d'initiation. J'essaie de faire en sorte qu'ils comprennent que la lumière n'est pas un accessoire ludique, même s'il est très ludique d'utiliser la lumière, mais qu'ils comprennent que la lumière est une composante de la scène qui, à part entière, toute seule, est déjà d'une extrême richesse spatio-temporelle. Je les incite aussi à se servir du moins de matériel possible pour expérimenter les choses le plus finement possible, de la manière la plus sensible.

Après, à partir de là, on fait des exercices très simples. Par exemple avec un objet et un projecteur (avant d'avoir une salle équipée j'utilisais une lampe de poche), de voir comment on peut raconter non pas une histoire, parce que je ne cherche pas à ce qu'il y ait un personnage ni à faire de l'anthropomorphisme, mais je voudrais simplement qu'il y ait un mini scénario en trois créations d'image. Mais ce n'est pas vraiment une image, parce qu'il faut qu'il y ait les effets, la transition entre les effets et réussir à construire un petit scénario de lumière. Donc, à grande échelle sur scène je les incite forcément à faire cela. Dans un deuxième temps, ou parallèlement, étant donné qu'ils sont en formation d'études théâtrales, je les incite à lire les textes de théâtre en ayant en tête des indications qui seraient celles pour la lumière et ensuite 
de se questionner sur la manière de dialoguer et de peut-être se détacher d'une lumière illustrative tout en comprenant qu'une dramaturgie de la lumière doit aller avec la dramaturgie de l'ensemble du spectacle.

\section{Costa - Quand avez-vous décidé d'être enseignante ?}

En fait, je viens d'une famille mixte d'artistes et d'enseignants. Ma mère était montessorienne, donc j'ai été élevée avec une méthode pédagogique très spécifique, où la part du sensible est très présente. Ma mère a été élève de Merleau-Ponty, c'està-dire de la phénoménologie de la perception. Elle a baigné dedans quand elle a été élève à la Sorbonne. Elle était née en 1924 et mes parents ont fait du théâtre dans les années 50 à Paris. J'ai été élevée dans une atmosphère très particulière, bien qu'à la montagne avec une certaine lumière... Avec des couchers de soleil magnifiques sur la neige en montagne, avec une forêt de feuillages d'automne et une lumière chatoyante dont je garde un souvenir étonnant. Et des soirées... La nuit, dans la forêt, mon frère aîné nous emmenait, quand j'étais petite, dans la forêt la nuit, en entendant les bruissements, en guettant la lumière de la lune... Excuse-moi pour cet épanchement lyrique, très personnel, mais pour expliquer que j'étais à la fois présupposée, je pense, à m'intéresser à la lumière sans le savoir, et puis avec une approche de l'enseignement que j'ai vécu, en tant qu'enfant, très particulière. Donc, je pense que j'avais en moi le désir de la transmission assez naturellement, et plus exactement du partage et de l'éveil. Je ne trouve rien de plus beau que de voir l'éveil de la sensibilité avec l'intelligence et la compréhension des choses chez quelqu'un dont on a la responsabilité de la formation. Donc quand j'ai cessé de faire de la lumière de spectacle, cette idée de la transmission est venue et aussi pour des raisons économiques, un moment donné il faut bien vivre.

Donc, mon mari étant aussi dans le spectacle, nous avons décidé que lui continuait. Nous avions deux enfants, c'était un peu plus logique à cette époque-là, et jai décidé d'être enseignante pour avoir le temps de gérer les choses domestiques. Néanmoins, j'essayais de faire les deux en même temps, c'était quand même très compliqué. Le week-end je voulais faire la lumière des spectacles de musique de mes frères. C'était encore possible, mais c'était de plus en plus occasionnel et j'ai eu la chance qu'on me propose, au bout d'un moment, un poste d'enseignement de théâtre. Donc j'ai enseigné uniquement le théâtre et l'histoire de l'art au lycée et j'ai fait construire un théâtre, une boîte noire. J'ai tout de suite fait installer la lumière et j'ai pu transmettre la lumière d'abord pour les élèves du lycée, et comme je faisais une thèse à ce moment-là sous la direction de Georges Banu à la Sorbonne Nouvelle à Paris 3, j'ai ensuite fait des concours et j'ai été recrutée à l'Université de Lille parce que j'avais les compétences pour transmettre cet enseignement-là et celui sur l'analyse du spectacle, particulièrement. Puis je suis devenue professeure et je dirige des recherches et un programme de recherche LdS « Lumière de Spectacle ».

\section{Faleiro - Donc vous avez travaillé dans ce lycée avec de l'art. Est-ce que vous pouvez expliquer comment ça se passe?}


Alors, je vais vous dire comment cela se passait, parce que depuis que j'ai cessé d'enseigner au lycée, il y a dix ans, il y a eu une première réforme, et maintenant il y en a une deuxième. L'art est toujours présent, l'enseignement artistique est toujours présent, il n'a pas été effacé des programmes, mais j'ai vu la transition se faire. C'est-àdire qu'en 1984/85 ont été créées les premières classes de spécialité théâtre. Ce sont des élèves qui avaient une formation de cinq heures de théâtre et qui le présentent au baccalauréat. C'était une matière forte, donc les étudiants devaient présenter une scène de théâtre et un dossier sur un programme national et qui était étudié pendant l'année avec leurs enseignants. Par exemple, la pièce Hamlet mise en scène par différents metteurs en scène, les différentes manières, dramaturgies et scénographies de lire et de mettre en scène Hamlet. Et à côté ils avaient la possibilité de prendre trois heures d'option facultative.

Donc moi, mes élèves ils avaient huit heures de théâtre par semaine, et en plus j'étais dans un lycée spécifique où nous leur offrions la possibilité d'avoir trois heures d'histoire de l'art. Donc ils étaient très artistes dans ce lycée, ils avaient du théâtre, du cinéma et des arts plastiques. Puis j'ai changé de lycée, et là c'était uniquement le théâtre, mais toujours les huit heures. C'était un groupe d'une douzaine d'élèves que je suivais pendant trois ans, de la classe de seconde à la terminale. Le niveau de formation auquel je les ai amenés était très élevé. Tellement élevé que, quand je suis arrivée à l'université et que j'ai eu 150 étudiants en première année, dont seulement un petit pourcentage avait fait ce type de classe de spécialités (les autres ont le droit de s'inscrire à l'université, c'est très libre, mais ne connaissent rien au théâtre), j'ai dû composer devant 150 étudiants, baisser le niveau et j'ai mis beaucoup de temps à retrouver la manière d'arriver au même niveau.

Ça m'a beaucoup surprise au début et maintenant, je l'ai vu, il y a eu là ce que j'appelais à l'époque " une démocratisation de l'enseignement du théâtre », avec la fermeture progressive des spécialités dans les lycées, mais davantage d'ouverture d'options de seulement trois heures. C'était plus démocratique pour l'accès à la culture, mais j'ai parlé aussi de dilution parce qu'il y avait une perte qualitative, et donc j'ai commencé à voir arriver aux examens du baccalauréat des élèves plus nombreux mais moins bien formés qualitativement. Je ne sais pas ce s'il faut regretter ou pas, mais je constate cela.

Voyons ce que le prochain baccalauréat va proposer à ce niveau-là. Et à l'université j'ai vu ça: plus démocratique mais une baisse de niveau. Donc, nous sommes là pour faire monter le niveau en trois ans de licence - et encore mieux après - en cinq ans (en ajoutant les deux ans de master), mais il est vrai que ça demande de l'énergie et du travail.

\section{Faleiro - Et au lycée, pendant ces 8 heures, y avait-il des moments de travail d'improvisation, de travail pratique, ou des lectures?}

Oui. Merci de poser la question, parce que c'est une donnée très spécifique à la France et c'est pourquoi on est en train de changer cela avec la réforme : parce que ça coûte cher à l'État. Sur les cinq heures, il y avait trois heures de pratique faites par un professionnel de pratique de jeu, en présence de l'enseignant. C'est-à-dire que 
nous sommes deux présents pour une douzaine d'élèves, payés pour faire les cours. Donc je comprends que cela coûte cher, mais c'était extrêmement précieux. Pourquoi avait-on besoin d'un artiste, contrairement à l'enseignement de la musique et à l'enseignement des arts plastiques, où on n'a pas besoin d'artiste puisque c'est l'enseignant qui a la charge de la totalité de la formation ? Parce qu'il n'y a pas de CAPES (Certificat d'aptitude professionnelle d'enseignement secondaire) ni d'agrégation de théâtre en France. On n'a pas de CAPES pour le théâtre; donc, on estime qu'on a besoin d'un professionnel. C'est d'une très grande richesse et j'ai fait ce métier pendant douze ans. J'ai eu plus d'une vingtaine de professionnels qui sont venus dans mes cours de théâtre et j'ai beaucoup aimé cette articulation: trouver ma place par rapport à lui, travailler ensemble... Nous avons fait une quantité de spectacles avec les étudiants en douze ans, quantité qui est très importante.

\section{Costa - Et est-ce que le désir de travailler le noir au théâtre vient de vos promenades au soir dans la forêt?}

Et bien, c'est un peu tout ça. Mais je dois dire qu'il y a trois Véronique en moi qui se sont intéressées à la lumière, peut-être même quatre. Il y a certainement la petite Véronique enfant. J'ai toujours aimé les atmosphères de clair-obscur, j'aime être dans la maison avec les rideaux tirés, je n'ai jamais aimé les plafonniers très éclairants, et j'aimais aussi, parce que j'habitais dans un chalet à la montagne, les coupures de courant où nous utilisions les bougies pour nous éclairer, et même sans ces raisons-là. J'ai perdu mon père quand j'étais très jeune et ma mère nous proposait des soirées uniquement à la bougie. Mon oncle pensait que c'était pour faire des économies, alors que c'était pour l'atmosphère. En hiver, le matin, quand je me levais pour le petit-déjeuner et qu'il faisait encore nuit, pour ne pas nous éblouir avec la lumière électrique, souvent il y avait une bougie sur la table du petit-déjeuner. Donc, je pense qu'il y a effectivement cette imprégnation d'enfant.

Ensuite, quand j'ai fait de la lumière dans les années 80 , j'ai travaillé sur un spectacle qui s'appelait Le Festin de l'ombre (1985), une adaptation d'un texte de Victor Hugo ; et Le Festin de l'ombre, comme son nom l'indique, m'inspirait beaucoup. Avec le scénographe, nous étions très inspirés par les clairs-obscurs et ce qu'on appelle les peintures au lavis où les peintres peignaient même au marc de café, dans des tonalités chaudes. C'était une spécialité de Victor Hugo. Et puis toute la poésie, tout l'univers de Victor Hugo est chargé de cette opposition et ce dialogue entre l'ombre et la lumière. Avec tout cela, il me paraissait évident d'utiliser le noir et la pénombre. Or, à chaque répétition, quand nous commencions la première scène... Donc, je mettais dans le noir, je commençais petit à petit et là j'improvisais. Il n'y avait pas de mémoire pour enregistrer les effets lumineux, je les faisais manuellement et j'étais en vibration avec ce qui se passait sur le plateau, avec ce que j'entendais, pour monter petit à petit, et je me fiais à mon œil. Je les maintenais comme ça dans quelque chose qui était une présence insensible de la lumière. Et au bout d'un moment, je dois le reconnaître, deux acteurs hommes, les plus âgés, disaient: "Mais ça suffit, on n'y voit rien. Qu'estce que c'est ? Ce n'est pas de la lumière !" Donc, ils étaient très en colère, ils avaient peur de tomber... Ce que je peux comprendre. Ils n'étaient pas du tout en accord 
avec cette proposition et je suis restée sur cet échec. Je me suis dit : "C'est vraiment dommage, on peut faire des choses tellement magnifiques en travaillant le noir !" Et ça c'était l'éclairagiste.

Il y avait l'enfant, l'éclairagiste et puis il y a eu la spectatrice, parce que j'ai toujours été très sensible à ces moments de noir. Notamment j'ai vu, dans les années 1980-1990, une évolution des intermèdes et des entractes, avec le passage à l'entracte, où les changements se font sans que le rideau soit baissé, dans un noir total où les techniciens doivent habituer leur oeil, pour pouvoir voir dans ce noir, avant que les spectateurs aient la même habitude que lui - donc, le plus rapidement possible afin que les spectateurs ne voient rien dans l'aménagement du plateau. Ce n'était pas un changement de décor absolu, mais plutôt bouger une table, une chaise, sortir un accessoire etc. Et puis j'ai vu progressivement qu'on a mis une forme de pénombre de façon à voir quand même les techniciens qui devenaient un peu joueurs avec la situation, et on a même fini par scénariser ces moments avec de la musique, mais tout en mettant une lumière spécifique qui montre bien qu'on est en train de changer de décor. J'ai vu cette progression se faire, à tel point qu'on appelle ça "une prime de feu". C'est-à-dire que le technicien avait une prime financière parce qu'il était vu sur le plateau. J'ai vu ce genre d'évolution, mais je me suis aussi intéressée au sens en me demandant comment représenter l'aveuglement sur scène. Dans une pièce où les gens sont aveugles, est-ce qu'on fait ressentir l'aveuglement aux spectateurs en les mettant dans une situation inconfortable de ne pas voir, ou quasiment pas, ou est-ce qu'on représente, en imitation, un personnage qui tatônne pour se déplacer et tout le monde comprend que cette personne est aveugle ? Alors, comment on implique le spectateur dans cette perception et quel est le point de vue qu'on veut adopter, celui de l'aveugle ou celui qui regarde l'aveugle ? Donc, j'ai commencé ce genre de réflexions et puis j'ai vu de plus en plus des spectacles qui ont utilisé l'obscurcissement, des moments très beaux et qui étaient toujours les plus poétiques pour moi, et je regrettais même parfois le moment où on mettait le plein feu.

Le questionnement était celui d'une spectatrice qui est devenu celui d'une chercheuse qui s'est vraiment intéressée à la question. J'ai commencé à écrire mes réflexions et à vouloir voir d'où venait le noir. Il est dit dans la Genèse et d'autres mythologies anciennes, y compris égyptienne, qu'on avait d'abord la nuit et ensuite la lumière. Effectivement on dit cela. Au théâtre, c'est la même chose, sauf qu'on a chassé le noir pour mettre de plus en plus de lumière. Ensuite, on ne savait plus refaire le noir. Avec l'éclairage à la chandelle ou au gaz, on ne savait pas éteindre et rallumer. Donc, comment on a retrouvé le noir et quelles en ont été les conditions, [c 'est] dont je parle aussi dans mon livre.

\section{Faleiro - Et sur la question du genre et du rapport à la lumière ? Êtes-vous, en France, en train de discuter de ce sujet?}

Oui. En trois mots. Dans le milieu du théâtre c'est très présent, parce que la place des femmes metteur en scène n'est absolument pas reconnue, car elle est souvent reléguée au théâtre Jeune Public. Il y a beaucoup de difficultés. Donc maintenant on 
est obligés de la considérer, et c'est même dans la loi. La loi de la parité, ça s'appelle. C'est obligatoire maintenant, avec tous les a priori que cela entraîne. "On a été obligés de prendre une femme, même si elle n'est pas bonne": on a encore entendu il n'y a pas si longtemps ce genre de choses, mais ça y est, ce genre de propos stupides commence à diminuer. On ne s'est jamais posé la question si on prenait des hommes mauvais comme directeurs de théâtre.

Ensuite, la deuxième chose c'est dans les études de genre. Dans les études théâtrales et universitaires c'est très présent. En France, on a beaucoup d'éclairagistes femmes et on commence à les connaître. Moi, quand je faisais de la lumière, j'ai aussi travaillé dans un théâtre où des spectacles venaient de la France entière, et chaque fois que le technicien de la lumière arrivait, il était sidéré de voir une femme et disait : "C'est la première fois que je vois une femme." En plus, j'avais un bébé. Ils disaient : "Mais comment tu fais ? Ce n'est pas possible !" Ils avaient beaucoup de mal à le penser, et à le croire aussi. Ce n'était pas facile. Mais elles étaient beaucoup plus qu'on ne le savait, comme dans beaucoup d'autres domaines, et on découvre petit à petit les noms de ces personnes. Maintenant elles sont, dans la formation des jeunes, presque plus nombreuses que les hommes. À l'ENSATT elles sont toujours plus nombreuses que les hommes.

Faleiro et Costa - Bien. Je crois que nous sommes plus que satisfaits. Merci beaucoup.

Je vous en prie, et merci pour cette invitation.

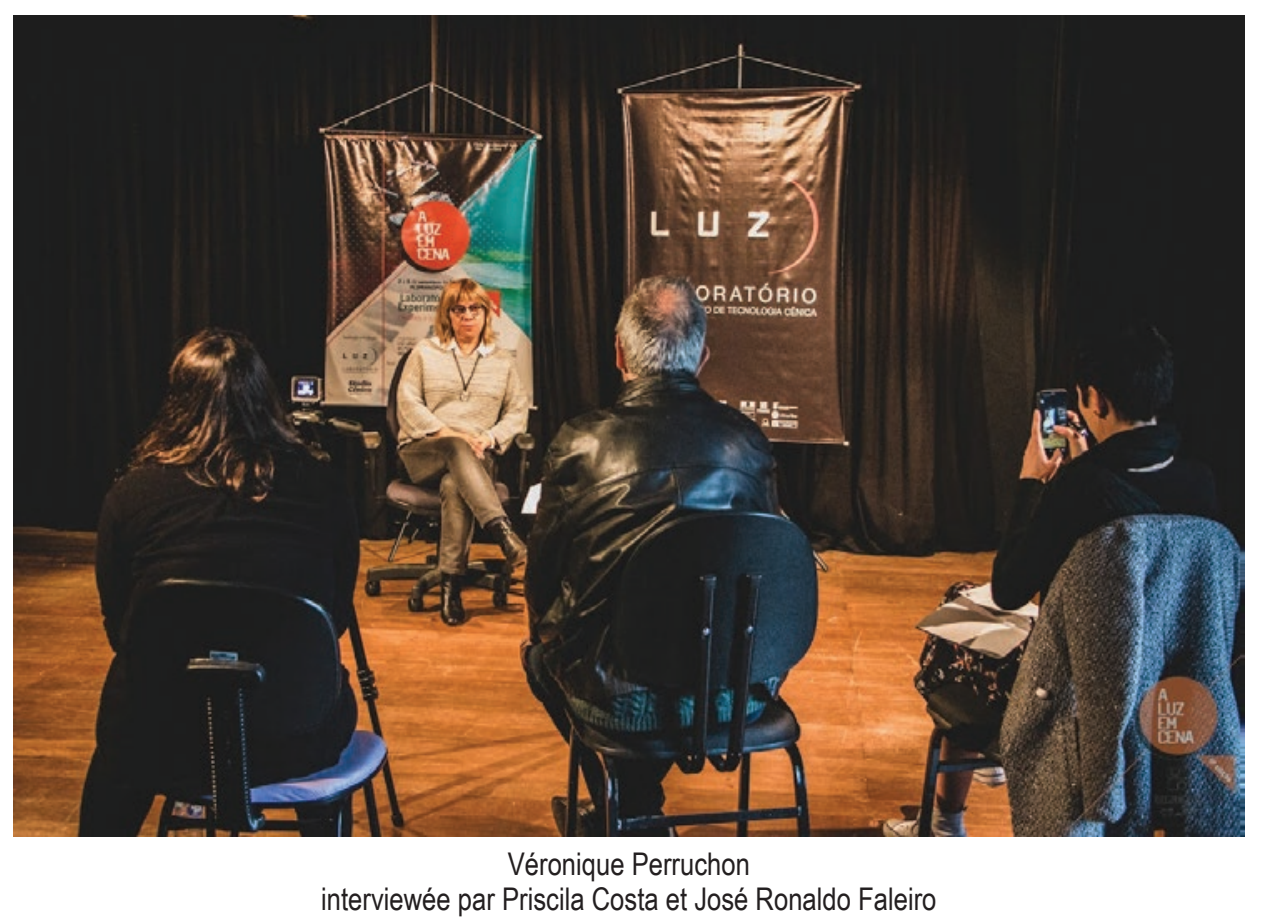

Reçu : 17/03/2020

Approuvé : 17/03/2020 